\title{
Effects of Contralateral Routing of Signal Hearing Aids on Audiological and Academic Performance in School-Age Children With Unilateral Hearing Loss
}

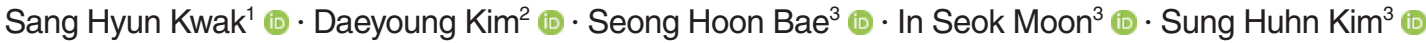 \\ Jae Young Choi ${ }^{3}$ (D) $\cdot$ Jinsei Jung ${ }^{3}$ (D) \\ ${ }^{1}$ Department of Otorhinolaryngology, St. Vincent's Hospital, College of Medicine, The Catholic University of Korea, Seoul; ${ }^{2}$ Research Center of \\ GN Hearing Korea, Seoul; ${ }^{3}$ Department of Otorhinolaryngology, Yonsei University College of Medicine and Brain Korea 21 PLUS Project for \\ Medical Sciences, Seoul, Korea
}

The recent introduction of newborn hearing screening programs for the early detection of unilateral hearing loss (UHL) has received increasing attention. In the 1980s, studies revealed that patients with UHL have increased risks for difficulties in language-related and other development areas [1,2]. In addition, UHL may increase behavioral problems and lead to lower academic achievement [3]. A common nonsurgical option for patients with UHL is to use a contralateral routing of signal (CROS) system [4]. Kenworthy et al. [5] showed that CROS systems can be useful in specific classroom settings by reporting positive audiological outcomes. To date, few studies have evaluated school performance-related auditory behaviors following auditory rehabilitation in school-age patients with UHL, because of the issue of compliance with the use of hearing aids (HAs). Herein, we prospectively assessed the effects of CROS HAs on schoolage participants with UHL, focusing on audiological outcomes and auditory behaviors through the use of related questionnaires.

A total of eight participants, comprising five female and three male schoolchildren with UHL, were prospectively enrolled. Their characteristics are shown in Supplementary Table 1. The participants ranged in age from 7 to 13 years (mean age, 10.2 \pm 1.8 years). The average hearing threshold in the affected ear was $95.9 \pm 21.8 \mathrm{~dB}$ HL. Most showed severe or profound hearing loss, except for one participant who had an average hearing threshold of $58.75 \mathrm{~dB}$ HL. All of the contralateral ears displayed normal

\footnotetext{
- Received December 28, 2020

Revised February 8, 2021

Accepted February 9, 2021

- Corresponding author: Jinsei Jung

Department of Otorhinolaryngology, Yonsei University College of Medicine,

50-1 Yonsei-ro, Seodaemun-gu, Seoul 03722, Korea

Tel: +82-2228-3622, Fax: +82-2-393-0580

E-mail: jsjung@yuhs.ac
}

hearing (6.6 $\pm 7.4 \mathrm{~dB} \mathrm{HL})$. This study was conducted in accordance with the recommendations of the Institutional Ethics Committee (No. 4-2017-1541). Written informed consent was obtained from the legal guardians of all minors and children in this study at the time of enrollment.

ReSound LiNX 3D LT562-DRW Receiver-In-Ear HAs with 12 channels were employed in the better-functioning ears, and SureFit CROS transmitters with wires were placed in the poorerfunctioning ears of the study participants (GN Hearing A/S, Ballerup, Denmark). The CROS HAs were fitted by experienced audiologists using the ReSound Aventa fitting program, as recommended by the manufacturer. The mean daily use time of the CROS HAs was $3.5 \pm 2.2$ hours.

All participants underwent pure-tone and speech audiometry, sound localization assessment, the Korean version of the Hearing in Noise Test (K-HINT), and behavioral tests using questionnaires prior to being fitted with CROS HAs. Follow-up testing was conducted 3, 6, and 12 months later. The questionnaire of the Korea National Institute for the Special Education-Auditory Behavioral Checklist (KNISE-ABC) was used to measure educational performance in school (Supplementary Material 1). The KNISE-ABC is composed of seven subcategories of questions covering listening, listening in background noise, communication, auditory attention, auditory memory, learning, and related behaviors.

Audiological outcomes and the results of auditory behavior testing were compared and analyzed with repeated-measures one-way analysis of variance (ANOVA). The Tukey correction was applied for the post-hoc analysis of between-group and within-group comparisons. Data are presented as mean \pm standard deviation. Differences between the mean values of groups were considered statistically significant at $P<0.05$.

Copyright (C) 2021 by Korean Society of Otorhinolaryngology-Head and Neck Surgery.

This is an open-access article distributed under the terms of the Creative Commons Attribution Non-Commercial License (https://creativecommons.org/licenses/by-nc/4.0) which permits unrestricted non-commercial use, distribution, and reproduction in any medium, provided the original work is properly cited. 


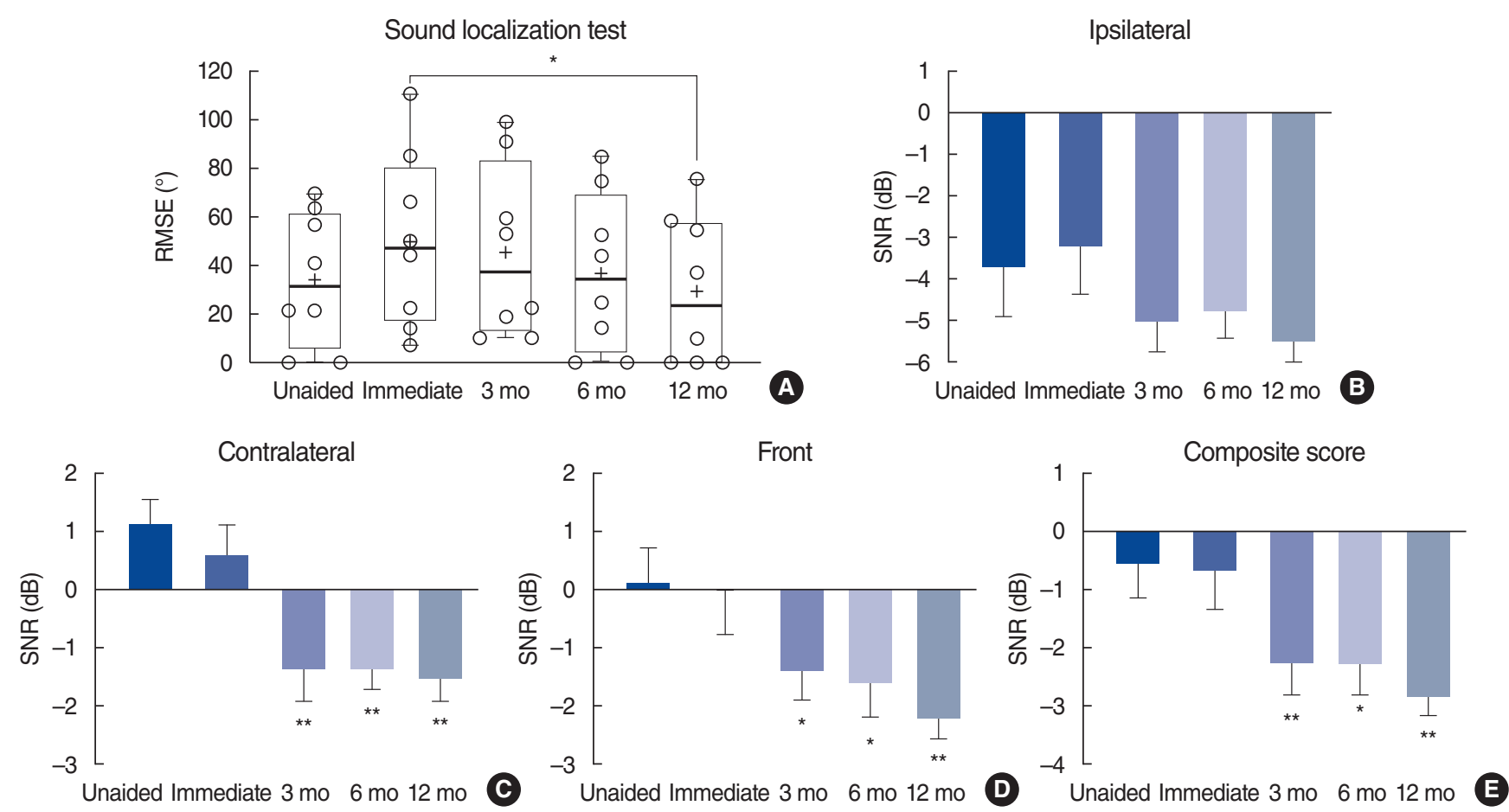

Fig. 1. (A) Outcomes of sound localization testing after contralateral routing of signal (CROS) hearing aid (HA) fitting in children with unilateral hearing loss (UHL). The ability of sound localization was measured as the root-mean-square error (RMSE) degree. The test was assessed at initial HA fitting (in both the unaided and CROS HA-aided states) and at 3, 6, and 12 months after HA fitting. (B-E) Outcomes of the Korean version of the Hearing in Noise Test (K-HINT) after CROS HA use in children with UHL. The composite scores were calculated using the formula: $[2 \times$ noise front+noise right+noise left]/4. Speech perception ability was evaluated in three types of noise environments (ipsilateral side, contralateral side, and front) in both the unaided and CROS HA-aided states. The K-HINT was administered at the initial HA fitting (in both the unaided and CROS HA-aided states) at 3, 6, and 12 months after HA fitting. SNR, signal-to-noise ratio. ${ }^{\star} P<0.05,{ }^{\star *} P<0.01$.

The serial root-mean-square error (RMSE) results, including those in the unaided state, are shown in Fig. 1A. Repeated-measures ANOVA revealed that the RMSE differed significantly between the immediate CROS-aided state $\left(49.8^{\circ} \pm 35.8^{\circ}\right)$ and the CROS-aided state at 12 months $\left(29.3^{\circ} \pm 30.6^{\circ}, P=0.01\right)$. Initially, the CROS-aided state seemed to yield a higher RMSE degrees than did the unaided state; however, no significant difference was observed between the two. We analyzed improvements in speech perception under noisy conditions using the K-HINT. When noise was delivered from the ipsilateral side (the deaf side), there were no improvements in signal-to-noise ratio (SNR) between the unaided and CROS-aided states at any point (Fig. 1B). In contrast, SNR showed significant enhancement when noise was delivered from the contralateral and front positions in the CROS-aided state compared with the unaided state at 3,6 , and 12 months after fitting the CROS HAs $(P<0.05)$ (Fig. $1 \mathrm{C}$ and $D)$. In addition, composite scores collected at 3,6 , and 12 months after fitting the CROS HAs were significantly better than those collected in the unaided state $(P<0.01)$ (Fig. 1E). Individual changes in K-HINT outcomes after CROS HA use are shown in Supplementary Fig. 1.

The obtained KNISE-ABC scores were compared with those of 1,045 school-age children (mean age, $9.1 \pm 2.1$ years; age range, 5-12 years; 518 male and 517 female) with normal hearing and
18 cochlear implant $(\mathrm{CI})$ patients (12 bilaterally implanted and 6 unilaterally implanted, 11 male and 7 female) with congenital hearing loss among school-aged children (mean age, 10.5 \pm 2.0 years) surveyed in prior published studies (Fig. 2, Supplementary Table 2) [6,7]. In the untreated state, all sections except learning garnered lower test scores than those of school-age children with normal hearing. In addition, the KNISE-ABC results were similar to those of $\mathrm{CI}$ patients in all sections except learning. However, after using the CROS HAs, the scores for listening in background noise at 6 months and communication at 12 months improved relative to those of $\mathrm{CI}$ patients. The scores for related behaviors recorded in the unaided state were poorer than those of the schoolchildren with CIs; however, after fitting the CROS HAs, this difference disappeared. After 12 months of using the CROS HAs, the recorded scores exhibited no differences between the normal school-age children and our participants in auditory attention, auditory memory, and learning. Since all CIs in the previous study were bilateral systems, the better outcomes of the KNISE-ABC in children with UHL are striking. However, there were no significant differences between the unaided state and the use of CROS-HA; only related behavior after 6 months of CROS-HA use was better than in the unaided state.

Some studies have reported that CROS HAs had a negative 

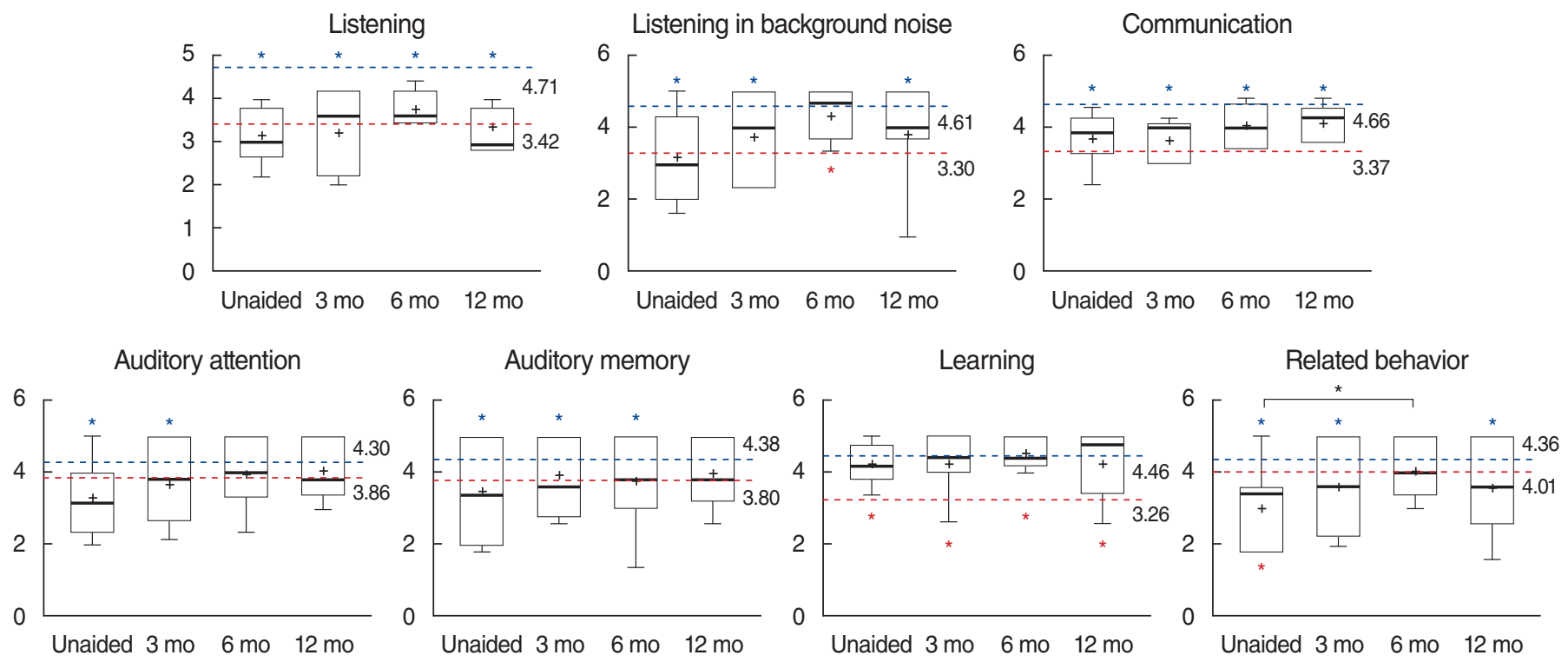

Fig. 2. Evaluation of school performance after contralateral routing of signal (CROS) hearing aid (HA) use in children with unilateral hearing Ioss (UHL). To evaluate school performance after CROS HA fitting, the Korea National Institute for the Special Education-Auditory Behavioral Checklist (KNISE-ABC) questionnaire was used. The KNISE-ABC consists of seven test areas: listening, listening in background noise, communication, learning, auditory memory, auditory attention, and related behaviors. The blue line indicates mean test results for schoolchildren with normal hearing [6]. The red line indicates the results for 18 elementary schoolchildren with cochlear implants (Cls) [7]. The red asterisks indicate statistical significance between $\mathrm{Cl}$ and CROS HA users $(P<0.05)$. The blue asterisks indicate statistical significance between schoolchildren with normal hearing and CROS HA users $(P<0.05)$. + refers to the mean scores of study participants.

effect on localization abilities, disrupting the monaural level and spectral cues for localization in the horizontal plane [8,9]. However, our results indicated no difference between unaided and aided states, in agreement with many other studies [10]. In fact, after 12 months of using the CROS HAs, RMSE scores were significantly better than those recorded immediately after the onset of CROS HA use. It seems that the participants became increasingly comfortable and well-adapted to using CROS HAs over the 12 months of the study.This may be attributable to the young age and high auditory plasticity of the participants. In summary, CROS HAs represent a nonsurgical intervention with the potential to improve school performance in children with UHL, encouraging efforts for the rehabilitation of children with UHL.

\section{CONFLICT OF INTEREST}

Sung Huhn Kim is an associate editor of the journal but was not involved in the peer reviewer selection, evaluation, or decision process of this article. No other potential conflicts of interest relevant to this article were reported.

\section{ACKNOWLEDGMENTS}

The authors would like to thank GN ReSound, Korea for scientific discussions about this project. This research was supported by the Research Program through the National Research Foundation of Korea (NRF) funded by the Korea government (2020R111A1A0107397611).

\section{ORCID}

Sang Hyun Kwak https://orcid.org/0000-0002-2827-8456

Daeyoung Kim https://orcid.org/0000-0002-0414-6352

Seong Hoon Bae https://orcid.org/0000-0001-9243-9392

In Seok Moon https://orcid.org/0000-0002-3951-5074

Sung Huhn Kim https://orcid.org/0000-0003-4408-3066

Jae Young Choi https://orcid.org/0000-0001-9493-3458

Jinsei Jung https://orcid.org/0000-0003-1906-6969

\section{AUTHOR CONTRIBUTIONS}

Conceptualization: JJ. Data curation: SHB. Methodology: DK. Project administration: ISM, JYC, SHK. Writing-original draft: SHK. Writing-reviewing \& editing: JJ.

\section{SUPPLEMENTARY MATERIALS}

Supplementary materials can be found via https://doi.org/10. 21053/ceo.2020.02523. 


\section{REFERENCES}

1. Bess FH, Tharpe AM. Unilateral hearing impairment in children. Pediatrics. 1984 Aug;74(2):206-16

2. Bess FH, Klee T, Culbertson JL. Identification, assessment, and management of children with unilateral sensorineural hearing loss. Ear Hear. 1986 Feb;7(1):43-51.

3. Culbertson JL, Gilbert LE. Children with unilateral sensorineural hearing loss: cognitive, academic, and social development. Ear Hear 1986 Feb;7(1):38-42.

4. Gordon KA, Papsin BC. Special edition on unilateral deafness and hearing loss: an introduction and overview. Hear Res. 2019 Feb;372: $1-2$.

5. Kenworthy OT, Klee T, Tharpe AM. Speech recognition ability of children with unilateral sensorineural hearing loss as a function of amplification, speech stimuli and listening condition. Ear Hear. 1990 Aug;11(4):264-70 .
6. Jang H, Kim-Lee Y, Lee H, Song Y. A study on the development of KNISE-auditory behavioral checklist. 1 ed. Asan: Korea National Institute for Special Education; 2012. p. 141.

7. Jang H, Yoon K, Ryu H. Auditory behavior characteristics of the students with cochlear implants in mainstream settings. J Incl Educ. 2016;11(2):167-86.

8. Pedley AJ, Kitterick PT. Contralateral routing of signals disrupts monaural level and spectral cues to sound localisation on the horizontal plane. Hear Res. 2017 Sep;353:104-11.

9. Lin LM, Bowditch S, Anderson MJ, May B, Cox KM, Niparko JK. Amplification in the rehabilitation of unilateral deafness: speech in noise and directional hearing effects with bone-anchored hearing and contralateral routing of signal amplification. Otol Neurotol. 2006 Feb;27(2):172-82.

10. Kitterick PT, Smith SN, Lucas L. Hearing instruments for unilateral severe-to-profound sensorineural hearing loss in adults: a systematic review and meta-analysis. Ear Hear. 2016 Sep-Oct;37(5):495-507. 